\title{
Correspondence
}

\section{PROSPECT guideline for elective caesarean section: a reply}

We thank Dr. Marr [1] for his thorough reading of the PROSPECT guideline for elective caesarean section [2] and for his valuable comments. Dr Marr is correct in stating that the evidence supporting dexamethasone is not strong. However, according to PROSPECT methodology, the use of dexamethasone can be recommended [3]. Indeed, an intervention is recommended if two randomised controlled trials provide evidence of clinically significant improvements in pain scores. Further, the PROSPECT methodology also allows for recommending an intervention if the Working Group deems it appropriate, based on the balance between the positive effects on pain scores and potential side effects. The PROSPECT Working Group concluded that although the evidence for improved pain scores with intravenous (i.v.) dexamethasone is limited, it is clinically significant, and dexamethasone is a relatively safe and easy intervention. An additional benefit is that dexamethasone is a potent anti-emetic with prolonged duration of effect and is considered a critical component of an optimal anti-emetic prophylaxis regimen.

Our discussion section acknowledges the limitations of our methodology as well as the limitations of the included studies [2]. Of note, we can only use the available published evidence for critical analyses. As an example, many interventions are studied where the comparison group received either saline or a placebo, without the administration of basic analgesia such as paracetamol and non-steroidal anti-inflammatory drugs or cyclooxygenase-2 specific inhibitors, which does not reflect current clinical practice, making conclusions challenging.

The study by Shalu et al. [4] had the methodological flaw of not administering basic analgesia. However, the duration of analgesia and the time to receive rescue analgesia were prolonged with i.v. dexamethasone. Further, pain scores for $24 \mathrm{~h}$ were clinically and statistically improved with i.v. dexamethasone. Dr Marr is correct that overall opioid consumption was not reduced in the study by Ituk et al. [5]. However, it was reduced in the 6-12-h interval. Additionally, this study also reported improved pain scores, both at rest and movement, at $12 \mathrm{~h}$ postoperatively. In fact, i.v. dexamethasone provided improvement in pain scores, although minimal, even with administration of intrathecal morphine. We want to emphasise that no other intervention studied reduced pain scores when intrathecal morphine was used. Maged et al. [6] showed weak evidence, especially in the local dexamethasone group, for better pain scores and reduced opioid consumption. So basically, we have two positive studies for dexamethasone and one study that displays limited positive effects.

The study by Edomwonyi et al. [7] was not included in our clinical recommendation since the comparator group included tramadol and not placebo.

In essence, we agree with Dr Marr when he states "None of these referenced publications display strong evidence that i.v. dexamethasone should be given to all women having elective caesarean section under spinal anaesthesia in order to improve their analgesia." Indeed the evidence is limited, but, together with the additional positive effects, the PROSPECT group concluded that it was appropriate to recommend i.v. dexamethasone.

\section{Acknowledgements}

No competing interests declared.

\section{E. Roofthooft}

GZA Sint-Augustinus Hospital, Antwerp, Belgium

\section{G.P. Joshi}

University of Texas Southwestern Medical Center, Dallas, TX, USA

\section{N. Rawal}

Orebro University

Orebro, Sweden

\section{Van de Velde}

KULeuven and UZLeuven,

Leuven, Belgium

Email: marc.vandevelde@uzleuven.be

\section{References}

1. Marr R. PROSPECT guideline for elective caesarean section and the administration of dexamethasone. Anaesthesia 2021. Epub 15 April. https://doi.org/https://doi.org/10.1111/anae.15484.

2. Roofthooft E, Joshi GP, Rawal N, et al. PROSPECT guideline for elective caesarean section: updated systematic review and procedure-specific postoperative pain management recommendations. Anaesthesia 2021; 76: 665-80.

3. Joshi GP, Van de Velde M, Kehlet H, et al. Development of evidence-based recommendations for procedure-specific pain management: PROSPECT methodology. Anaesthesia 2019; 74: 1298-304.

4. Shalu PS, Ghodki PS. To study the efficacy of intravenous dexamethasone in prolonging the duration of spinal anesthesia 
in elective cesarean section. Anesthesia Essays and Researches 2017; 11: 321-5.

5. Ituk $U$, Thenuwara $K$. The effect of a single intraoperative dose of intravenous dexamethasone $8 \mathrm{mg}$ on post-cesarean delivery analgesia: a randomized controlled trial. International Journal of Obstetric Anesthesia 2018; 35: 57-63.

6. Maged AM, Deeb WS, Elbaradie S, et al. Comparison of local and intravenous dexamethasone on postoperative pain and recovery after caesarean section. A randomized controlled trial.
Taiwanese Journal of Obstetrics and Gynecology 2018; 57: 34650.

7. Edomwonyi NP, Osazuwa MO, Iribhogbe OI, Esangbedo SE. Postoperative analgesia using bupivacaine wound infiltration with intravenous tramadol or dexamethasone following obstetric spinal anaesthesia. Nigerian Journal of Clinical Practice 2019; 20: 1584-9.

doi:10.1111/anae.15496 